\title{
Cartilage degeneration is associated with activation of the PI3K/AKT signaling pathway in a growing rat experimental model of developmental trochlear dysplasia
}

\section{Wei Lin}

Hebei Medical University Third Affiliated Hospital

\section{Yike Dai}

Hebei Medical University Third Affiliated Hospital

Jinghui Niu

Hebei Medical University Third Affiliated Hospital

\section{Chongyi Fan}

Hebei Medical University Third Affiliated Hospital

\section{Xunkai Feng}

Hebei Medical University Third Affiliated Hospital

Fei Wang ( $\nabla$ doctorlinw@163.com)

Hebei Medical University Third Affiliated Hospital

\section{Research article}

Keywords: Trochlear dysplasia, Patellar instability, PI3K/AKT, Cartilage degeneration, Osteoarthritis

Posted Date: May 26th, 2020

DOl: https://doi.org/10.21203/rs.3.rs-24905/v2

License: (c) (i) This work is licensed under a Creative Commons Attribution 4.0 International License.

Read Full License

Version of Record: A version of this preprint was published at Journal of Advanced Research on April 1st, 2021. See the published version at https://doi.org/10.1016/j.jare.2021.04.006. 


\section{Abstract}

Background: Trochlear dysplasia is a commonly encountered lower extremity deformity in humans. However, the molecular mechanism of cartilage degeneration in trochlear dysplasia is unclear thus far. The PI3K/AKT signaling pathway is known to be important for regulating the pathophysiology of cartilage degeneration. This study investigated the relationship of the PI3K/AKT signaling pathway with trochlear dysplasia cartilage degeneration.

Materials and methods: In total, 120 female Sprague-Dawley rats (4 weeks of age) were randomly separated into control and experimental groups. Distal femurs were isolated from the experimental group at 4,8 , and 12 weeks after surgery; they were isolated from the control group at the same time points. Micro-computed tomography and histological examination were performed to investigate trochlear anatomy and changes in trochlear cartilage. Subsequently, expression patterns of $\mathrm{PI} 3 \mathrm{~K} / \mathrm{AKT}$, TGF $\beta 1$, and ADAMTS-4 in cartilage were investigated by immunohistochemistry and quantitative polymerase chain reaction.

Results: In the experimental group, the trochlear dysplasia model was successfully established at 8 weeks after surgery. Moreover, cartilage degeneration was observed beginning at 8 weeks after surgery, with higher protein and mRNA expression levels of PI3K/AKT, TGF $\beta 1$, and ADAMTS-4, relative to the control group.

Conclusions: Patellar instability might lead to trochlear dysplasia in growing rats. Moreover, trochlear dysplasia may cause patellofemoral osteoarthritis; cartilage degeneration in trochlear dysplasia might be associated with activation of the PI3K/AKT signaling pathway. These results provide insights regarding the high incidence of osteoarthritis in patients with trochlear dysplasia. However, more research is needed to clarify the underlying mechanisms.

\section{Introduction}

Trochlear dysplasia (TD) of the femur is a common deformity of the lower extremities; it is regarded as an abnormal anatomical morphology of the depth or angle of the medial or lateral groove facets [1]. In 1964, Brattström first proposed a relationship between TD and patellar instability [2]. Approximately $85 \%-90 \%$ of patients with patellar instability reportedly exhibit TD [3,4]. Consequently, many scholars thought that TD was a pathogenic factor of patellar instability $[5,6]$. Recent studies have shown that the development of TD might be related to excessive femur anteversion, patella alta, and greater tibial tubercle to trochlear groove distance [7-12]. In addition, TD can lead to patellar instability, patellar maltracking, and abnormal pressure load distribution; the most common long-term complication is osteoarthritis (OA) [13-14]. Some scholars consider TD to be a pathogenic factor of early patellofemoral OA [15]. To the best of our knowledge, there have been few studies regarding TD-induced cartilage degeneration at the molecular level. 
Articular cartilage has a very low matrix and cell turnover rate, because of its permanent nature $[16,17]$. Because chondrocytes comprise the only cell type in articular cartilage, an imbalance between chondrocyte proliferation and apoptosis is an important factor associated with onset of OA [18]. Consequently, maintenance of the balance between chondrocyte proliferation and apoptosis can reduce cartilage degeneration. Many signaling pathways are known to be involved in cartilage degeneration; regulation of the PI3K/AKT signaling pathway is an important contributor to the pathogenesis of cartilage degeneration, because of its key roles in several characteristic changes in cartilage (e.g., expression of aggrecanases [ADAMTS-4 and ADAMTS-5] and matrix metalloproteinases) [19]. Furthermore, some studies have shown that activation of the PI3K/AKT signaling pathway can promote osteogenic differentiation of pre-osteoblasts and mesenchymal stem cells; targeted inhibition of the $\mathrm{PI3K} / \mathrm{AKT}$ signaling pathway can induce bone loss and reduce bone formation [20-22]. Transforming growth factor $\beta 1$ (TGF $\beta 1$ ) is known to be an important contributor to chondrocyte development; it can regulate extracellular matrix biosynthesis and has been extensively studied as a regulator of cartilage metabolic activity [23]. Previous studies have shown that TD can cause articular cartilage degeneration $[24,25]$. However, the molecular mechanism of TD-induced cartilage degeneration has been uncertain thus far. Moreover, the effects of the PI3K/AKT signaling pathway in TD-induced cartilage degeneration have not been reported.

Here, to study the association of PI3K/AKT signaling with TD-induced cartilage degeneration, we investigated the expression pattern of the PI3K/AKT pathway in cartilage at different stages in a growing rat model of experimental TD by immunohistochemistry and quantitative real-time polymerase chain reaction (qPCR). We speculated that TD might lead to overexpression of PI3K/AKT, thus resulting in cartilage degeneration. To the best of our knowledge, this is the first study regarding the association between $\mathrm{PI} 3 \mathrm{~K} / \mathrm{AKT}$ and cartilage degeneration in a growing rat model of experimental TD.

\section{Materials And Methods}

\section{Study design}

The Animal Ethics Committee of the Third Hospital of Hebei Medical University approved this study (approval number: Z2019-005-1). Four-week-old female Sprague-Dawley rats were obtained from the Laboratory Animal Center of Hebei Medical University. Rats have been shown to require 12 weeks of bone development to reach a mature state [26]. In this study, 120 rats were randomly separated into control and experimental groups ( $\mathrm{n}=60$ per group). In the control group, the rats' left knees did not undergo any surgery. In the experimental group, the rats' left knees underwent surgery to induce patellar instability. Rats were sacrificed by overdose anesthesia at 4,8 , and 12 weeks after surgery. Left distal femur tissues were collected after surgery ( $n=20$ knees/time point in each group).

\section{Surgical technique}


In this study, pentobarbital sodium (30 mg/kg, intraperitoneal injection) was used to induce anesthesia in all rats; they were then shaved and disinfected. An incision was then made along the midline of the skin; the skin and subcutaneous tissue were separated, exposing the joint capsule through the medial approach to the knee. The procedure to induce patellar instability was performed as described in our previous studies [24, 25]. Specifically, a $0.5-\mathrm{cm}$ longitudinal incision was made along the patella at the medial capsule and retinaculum. In this manner, patellar instability could be observed during surgery. All procedures were carefully performed to avoid cartilage damage. The incision was carefully flushed, then sutured. Finally, the wound was bandaged. To control postoperative pain, rats were administered acetaminophen (30 mg/kg, daily) for 5 days.

\section{Macroscopic morphological and micro-computed tomography (CT) assessment}

All distal femurs were carefully harvested and scanned via micro-CT (SkyScan model 1076, SkyScan, Kontich, Belgium; parameters: $10 \mu \mathrm{m}$ voxel size at $50 \mathrm{kV}$ and $800 \mu \mathrm{A}$ ) at 4, 8, 12 week after surgery, respectively. As described previously [12, 24], axial slices of the trochlea were identified; trochlear depth and sulcus angle were then calculated (Figure 1). Using the diagnostic criteria defined by Dejour et al. [4], TD was diagnosed in micro-CT images.

For analysis of microstructural parameters, the region of interest was located transversely below the lateral and medial facets of the trochlea with two red cylinders of 3-mm diameter (Figure 1); micro-CT scanning data were transformed into a three-dimensional model by using Mimics software, version 19.0 (Materialise, Leuven, Belgium). Microstructural parameters analyzed in this study included bone volume to total volume fraction $(\%)$, trabecular number $(1 / \mathrm{mm})$, trabecular thickness $(\mathrm{mm})$, trabecular separation $(\mathrm{mm})$, and bone mineral density $\left(\mathrm{mg} / \mathrm{cm}^{3}\right)$.

\section{Histological staining}

Samples were isolated at each time point, fixed with $4 \%$ paraformaldehyde, decalcified in $10 \%$ ethylenediaminetetraacetic acid until completely demineralized, and embedded in paraffin. Fivemicrometer slices were cut along the femoral axis to obtain axial images of the trochlear groove. Glycosaminoglycans in cartilage were assessed using Safranin 0; evaluation of cartilage degradation was performed by fast green counterstaining of protein. The modified Mankin scale was used to determine the grade of cartilage degeneration [27].

\section{Immunohistochemistry}


Slices were deparaffinized in xylene and rehydrated. At room temperature, slices were washed three times with phosphate-buffered saline ( 5 minutes per wash). Endogenous peroxidase activity was blocked by incubation of slices in $3 \%$ hydrogen peroxide for 10 minutes. Antigen retrieval was performed by microwave treatment of slices for 10 minutes in $10 \mathrm{mM}$ sodium citrate $(\mathrm{pH}$ 6.0). Slices were incubated overnight at $4^{\circ} \mathrm{C}$ with anti-PI3K (BoAoSen, Beijing, China), anti-AKT (Servicebio, Wuhan, China), antiTGFB1 (BoAoSen), or anti-ADAMTS-4 (BoAoSen) primary antibodies at a dilution of 1:50. For the negative control, the primary antibody step was omitted. Subsequently, the objective magnification was adjusted to $20 \times 100$, five regions were randomly selected for all slices in each group, and the entire area of each region was imaged. During microscopy, the tissue covered the entire field of view; the background light was maintained at a consistent level. Image-Pro Plus 6.0 software (Media Cybernetics, Rockville, MD, USA) was used for analysis of all microscopy images. All images were analyzed to acquire data regarding cumulative optical density and tissue area. The areal density was defined as cumulative optical density divided by tissue area. The areal density value is positively correlated with positive protein expression.

\section{$q P C R$}

Samples were analyzed by qPCR to determine mRNA expression levels at 4,8 , and 12 weeks after surgery. Trizol reagent (Servicebio) was used to extract RNA from chondrocytes and cartilage. The RevertAid $^{\text {TM }}$ first-strand cDNA synthesis kit (Cat. No. K1622, Thermo Fisher Scientific, Waltham, MA, USA) was used for reverse transcription of mRNA into cDNA. Primers for PI3K, AKT, TGF 31 , and ADAMTS-4 were used, with a sequence detection system for gene analysis. mRNA expression of target genes was determined with reference to $G A P D H$ and calculated using the formula $2^{-\triangle \triangle C t}$ (cycle threshold method). All primers used in this study are listed in Table 1. Each experiment was performed three times and mean values were used for further analyses.

\section{Statistical analysis}

Mean and standard deviation were used for descriptive statistical analysis. The Shapiro-Wilk test was used to determine normality for each variable, while Levene's test was used to assess homogeneity of variance. Student's t-test was used for comparisons between two groups; one-way analysis of variance was used for comparisons among $\geq 3$ groups. SPSS Statistics, version 19.0 (IBM Corp., Armonk, NY, USA) was used for all statistical analyses. Differences with $p<0.05$ were considered statistically significant. Preliminary analysis suggested that at least six rats were needed at each time point and in each group, to achieve $80 \%$ efficacy $(1-\beta)$ and $90 \%$ confidence [28].

\section{Results}


The TD model was successfully established, characterized by a larger groove angle and flatter trochlear groove (Tables 2 and 3 ). The trochlear depth and sulcus angle did not exhibit significant differences between the control and experimental groups $(p>0.05)$ at 4 weeks after surgery; however, they exhibited significant differences between the two groups $(p<0.05)$ at 8 and 12 weeks after surgery. Over time, the trochlear groove became significantly flatter and sulcus angle became significantly larger, compared with the control group (Figure 2).

\section{Detection of subchondral bone loss by micro-CT}

In the experimental group, micro-CT displayed obvious loss of subchondral bone at 8 weeks after surgery, and became more and more serious with time. Micro-CT scans of the two groups revealed that the bone volume to total volume fraction, trabecular number, and trabecular thickness decreased markedly at 8 and 12 weeks after surgery $(p<0.05)$; moreover, trabecular separation significantly increased at 8 and 12 weeks after surgery $(p<0.05)$. However, bone mineral density did not remarkably differ $(p>0.05)$ (Figure $3)$.

\section{Loss of cartilage proteoglycans and higher Mankin score in the TD model}

At 4 weeks after surgery, no significant differences were present in cartilage appearance between the two groups. However, the amount of matrix proteoglycans was significantly reduced, the cartilage surface was damaged (accompanied by rougher and thinner appearance), and chondrocytes were disordered in the experimental group at 8 and 12 weeks after surgery (Figure 4). Moreover, Mankin scores were higher in the TD model cartilage at different stages and worsened over time (Figure 4$)(p<0.05)$.

\section{Increased expression of PI3KJAKT, TGFB1, and ADAMTS-4 in the TD model cartilage}

Immunohistochemistry analysis revealed that the expression of PI3K/AKT was slightly elevated at 4 weeks after surgery $(p<0.05)$, compared with the control group; however, the expression patterns of TGF $\beta 1$ and ADAMTS-4 showed no remarkable changes $(p>0.05)$. Additionally, there were remarkable differences in PI3K/AKT, TGF $\beta 1$, and ADAMTS-4 expression at 8 and 12 weeks after surgery $(p<0.05)$ (Figure 5,6$)$. Furthermore, qPCR analysis showed expression patterns similar to those of immunohistochemical analysis $(p<0.05)$ (Figure 7$)$.

\section{Discussion}


In this study, we successfully established an experimental model of TD in growing rats after induction of patellar instability; the model was characterized by subchondral bone loss and cartilage degeneration, which worsened over time. Importantly, we observed remarkably elevated expression of PI3K/AKT at the protein and mRNA levels in the cartilage degeneration model, which revealed that TD-induced cartilage degeneration might be associated with activation of the PI3K/AKT signaling pathway. These results provide new insights regarding the high incidence of OA in patients with TD. To the best of our knowledge, this is the first report regarding the relationship between PI3K/AKT signaling and cartilage degeneration in the patellar instability model of TD.

Mechanical stress is known as a major factor in the development of bone and cartilage [29]. Changes in stress distribution in the patellofemoral joint will affect cartilage metabolism; positional mismatches between the patella and femoral trochlea may lead to OA [30-32]. When patellofemoral joint pressure increases due to abnormal patellar biomechanics, cartilage is damaged more easily [33-34]. The present results support the hypothesis that TD may be induced by mechanical stimuli, because pressure changes in the patellofemoral joint due to patellar instability; such changes worsen over time during TD progression. However, mechanical factors may not be the only means by which TD develops into OA. Because TD is a developmental disease, abnormal joint development may lead to early articular cartilage degeneration.

The PI3K/AKT signaling pathway is a key component of cell development, including cell metabolism, apoptosis, transcription, and cell cycle [35]. Additionally, abnormal expression levels of PI3K/AKT have been found in many cancers [36]. Previous studies have shown that PI3K/AKT signaling contributes to the pathogenesis of OA [37,38]; inhibition of PI3K/AKT expression in rat chondrocytes can improve autophagy and delay the progression of OA $[39,40]$. The progression of OA is known to be affected by inflammatory cytokines, and the PI3K/AKT signaling pathway can mediate NF-kB activation and the mRNA expression of TNFa in osteoblasts; inhibition of PI3K/AKT signaling can protect chondrocytes from inflammatory disruption in OA [41-44]. Thus, we presumed that the PI3K/AKT signaling pathway plays an important role in TD pathology. To test this hypothesis, we examined PI3K/AKT expression in rat trochlear cartilage. Immunohistochemistry and mRNA expression analyses revealed that the levels of PI3K/AKT increased over time in the patellar instability model of TD. Thus, persistent high expression of $\mathrm{PISK} / \mathrm{AKT}$ in the TD model may explain the continued existence of developmental factors in articular cartilage. However, further studies are needed to clarify how PI3K/AKT contributes to TD pathology.

TGF $\beta 1$ is an important research focus, with potential for clinical use; it may aid in diagnosis and treatment of OA [45]. Abnormally elevated levels of TGF $\beta 1$ in cartilage can promote production of proteoglycans and lead to abnormal growth of osteophytes and synovium [46]. TGF $\beta 1$ accelerates the condensation of mesenchymal stem cells, and improves early chondrocyte differentiation, while inhibiting terminal hypertrophic differentiation; thus, it influences regular bone morphology [47]. In the present study, persistent high expression of TGF $\beta 1$ in the TD model increased in an age-dependent manner. Overall, these findings suggested that articular cartilage in the rat TD model exhibited early molecular expression of OA. 
Notably, our previous study confirmed that TD can lead to cartilage degeneration [24, 25]. In the present study, we found that, with increasing age, cartilage morphology changed in the rat TD model: its surface became rough, vertical cracks appeared, and articular chondrocytes aggregated. Furthermore, qPCR analysis revealed that ADAMTS-4 [48], a marker of cartilage degeneration, was overexpressed in cartilage of the TD model. Thus, this study confirmed the presence of cartilage degeneration in a model of TD. These findings were consistent with the manifestation of OA, suggesting that TD may contribute to onset of patellofemoral OA.

Our study had several limitations. First, findings in a rat model cannot be readily translated to the clinic. Second, this study only investigated the early stage of patellofemoral OA at 12 weeks after induction of patellar instability; it did not generate long-term follow-up data. Third, further investigation is needed regarding the molecular mechanisms of PI3K/AKT signaling during TD-induced cartilage degeneration.

\section{Conclusion}

The findings in this study suggested that patellar instability may lead to TD in growing rats. Moreover, TD may cause patellofemoral OA; TD-induced cartilage degeneration might be associated with activation of the PI3K/AKT signaling pathway. These results provide new insights regarding the high incidence of OA in patients with TD. However, additional research is needed to clarify the underlying mechanisms.

\section{Abbreviations}

TD: trochlear dysplasia; OA: osteoarthritis; qPCR: quantitative polymerase chain reaction; micro-CT: micro-computed tomography.

\section{Declarations}

\section{Ethics approval}

This study was approved by the Animal Ethics Committee of the Third Hospital of Hebei Medical University (approval number: Z2019-005-1).

\section{Consent for publication}

Not applicable.

\section{Availability of data and materials}


The detailed data and materials of this study are available from the corresponding author (via email) on reasonable request.

\section{Competing interests}

The authors declare that they have no competing interests.

\section{Funding}

This work was supported by the National Natural Science Foundation of China (grant no. 81873983), Key Program of Natural Science Foundation of Hebei Province (grant no. H2019206694), Hebei Province Key Project of Achievement Transformation (grant no. zh2018007), and the Fund for Graduates' Innovative Projects of the Academic Degree Office of Hebei Provincial Department of Education (grant no. CXZZBS2020114).

\section{Authors' contributions}

FW designed the study. WL, YKD, and JHN performed the experiments. CYF, XKF, and WL evaluated the data. WL wrote the manuscript. All authors read and approved the final manuscript.

\section{Acknowledgments}

The authors would like to thank the staff of all participating departments.

\section{References}

1. Sillanpää P, Mattila VM, livonen T, Visuri T, Pihlajamäki $H$. Incidence and risk factors of acute traumatic primary patellar dislocation. Med Sci Sports Exerc. 2008, 40(4):606-11.

2. BRATTSTROEM H. Shape of the Intercondylar Groove Normally and in Recurrent Dislocation of Patella: A Clinical and X-Ray Anatomical Investigation. Acta Orthop Scand Suppl. 1964, 68:SUPPL 68:1-148.

3. Fithian DC, Paxton EW, Cohen AB. Indications in the treatment of patellar instability. J Knee Surg. 2004, 17(1):47-56.

4. Dejour H, Walch G, Nove-Josserand L, Guier C. Factors of patellar instability: an anatomic radiographic study. Knee Surg Sports Traumatol Arthrosc.1994, 2(1):19-26. 
5. Laurin CA, Lévesque HP, Dussault R, Labelle H, Peides JP. The abnormal lateral patellofemoral angle: a diagnostic roentgenographic sign of recurrent patellar subluxation. J Bone Joint Surg Am. 1978, 60(1):55-60.

6. Goldthwait JE. Slipping or recurrent dislocation of the patella: with the report of eleven cases. J Bone Joint Surg Am. 2003, 85(12):2489.

7. Parikh SN, Rajdev N, Sun Q. The growth of trochlear dysplasia during adolescence. J Pediatr Orthop. 2018, 38(6):e318-e324.

8. Franciozi CE, Ambra LF, Luzo MV, et al. Increased femoral Anteversion influence over surgically treated recurrent patellar instability patients. Arthroscopy. 2017, 33(3):633-640.

9. Ferlic PW, Runer A, Dammerer D, Wansch J, Hackl W, Liebensteiner MC. Patella height correlates with trochlear dysplasia: a computed tomography image analysis. Arthroscopy. 2018, 34 (6): 1921-1928.

10. Williams AA, Elias JJ, Cosgarea AJ, et al. The relationship between Tibial tuberosity-trochlear groove distance and abnormal patellar tracking in patients with unilateral patellar instability. Arthroscopy. 2016, 32(1):55-61.

11. Dejour D, Le Coultre B. Osteotomies in Patellofemoral instabilities. Sports Med Arthrosc. 2018, 26(1):8-15.

12. Kang H, Lu J, Wang F, et al. The effect of increased femoral anteversion on the morphological and trabecular microarchitectural changes in the trochlea in an immature rabbit. J Adv Res. 2020, 23:143149.

13. Fitoussi F, Akouré S, Chouteau Y, Bouger D. Hollow femoral trochlea and femoro-patellar osteoarthritis. Rev Chir Orthop Reparatrice Appar Mot.1994, 80(6):520-4.

14. Dejour H, Walch G, Neyret P, Adeleine P. Dysplasia of the femoral trochlea. Rev Chir Orthop Reparatrice Appar Mot. 1990, 76(1):45-54.

15. Jungmann PM, Tham SC, Link TM, et al. Association of trochlear dysplasia with degenerative abnormalities in the knee: data from the osteoarthritis initiative. Skeletal Radiol. 2013, 42(10):138392.

16. Wu Q, Chen D, Zuscik MJ, O'Keefe RJ, Rosier RN. Overexpression of Smurf2 stimulates endochondral ossification through upregulation of $\beta$-catenin. J Bone Miner Res.2008, 23(4):552-63.

17. Wang D, Taboas JM, Tuan RS. PTHrP overexpression Partially inhibits a mechanical strain-induced arthritic phenotype in chondrocytes. Osteoarthritis Cartilage. 2011, 19 (2): 213-221.

18. Sezgin M, Barlas ÍÖ, Erdal ME, et al. Apoptosis-related Fas and FasL gene polymorphisms' associations with knee osteoarthritis. Rheumatol Int. 2013, 33(8):2039-43.

19. Huang $\mathrm{CY}$, Lin HJ, Chen HS, Cheng SY, Hsu HC, Tang CH. Thrombin Promotes matrix metalloproteinase-13 expression through the PKCdelta c-Src/EGFR/PI3K/Akt/AP-1 signaling pathway in human chondrocytes. Mediators Inflamm. 2013, 2013:326041.

20. Baker N, Sohn J, Tuan RS. Promotion of human mesenchymal stem cell osteogenesis by PI3kinase/Akt signaling, and the influence of caveolin-1/cholesterol homeostasis. Stem Cell Res Ther. 
2015,$01 ; 6: 238$.

21. Lauzon MA, Drevelle O, Daviau A, Faucheux N. Effects of BMP-9 and BMP-2 on the PI3K/Akt Pathway in MC3T3-E1 Preosteoblasts. Tissue Eng Part A. 2016, 22 (17-18): 1075- 1085.

22. Xi JC, Zang HY, Ma YZ, et al. The PI3K/AKT cell signaling pathway is involved in regulation of osteoporosis. J Recept Signal Transduct Res. 2015, 35(6):640-5.

23. Haudenschild DR, Chen J, Steklov N, Lotz MK, D'Lima DD. Characterization of the chondrocyte actin cytoskeleton in living three-dimensional culture: response to anabolic and catabolic stimuli. Mol Cell Biomech. 2009, 6(3):135-44.

24. Dai Y, Lu J, Li F, Yang G, Ji G, Wang, F. Changes in cartilage and subchondral bone in a growing rabbit experimental model of developmental trochlear dysplasia of the knee. Connect Tissue Res. 2019, $12 ; 1-14$.

25. Yang G, Li F, Wang F, et al. The dysplastic trochlear sulcus due to the insufficient patellar stress in growing rats. BMc Musculoskelet Disord. 2019, 05;20(1):411.

26. Chan EF, Harjanto R, Sah RL, et al. Structural and functional maturation of distal femoral cartilage and bone during postnatal development and growth in humans and mice. Orthop Clin North Am. 2012, 43(2):173-85,v.

27. Mankin HJ, Johnson ME, Lippiello L. Biochemical and metabolic abnormalities in articular cartilage from osteoarthritic human hips. III. Distribution and metabolism of amino sugar- containing macromolecules. J Bone Joint Surg Am.1981, 63(1):131-9.

28. Ranstam J. Repeated measurements, bilateral observations and pseudoreplicates, why does it matter? Osteoarthritis Cartilage. 2012, 20(6):473-5.

29. Guevara JM, Moncayo MA, Vaca-González JJ, Gutiérrez ML, Barrera LA, Garzón-Alvarado DA. Growth plate stress distribution implications during bone development: A simple framework computational approach. Comput Methods Programs Biomed. 2015, 118(1 ):59-68.

30. Fujikawa K, Seedhom BB, Wright V. Biomechanics of the patellofemoral joint. Part II: a study of the effect of simulated femorotibial varus deformity on the congruity of the patellofemoral compartment and movement of the patella. Eng Med.1983, 12(1):13-21.

31. Harrison MM, Cooke TD, Fisher SB, Griffin MP. Patterns of knee arthrosis and patellar subluxation. Clin Orthop Relat Res.1994, (309):56-63.

32. Iwano T, Kurosawa H, Tokuyama H, Hoshikawa Y. Roentgenographic and clinical findings of patellofemoral osteoarthrosis. With special reference to its relationship to femorotibial osteoarthrosis and etiologic factors. Clin Orthop Relat Res. 1990, (252):190-7.

33. Grelsamer RP, Weinstein CH. Applied biomechanics of the patella. Clin Orthop Relat Res. 2001, (389):9-14.

34. Post WR, Teitge R, Amis A. Patellofemoral malalignment: looking beyond the viewbox. Clin Sports Med. 2002, 21(3):521-46, x. 
35. Malemud CJ. The PI3K/Akt/PTEN/mTOR pathway: a fruitful target for inducing cell death in rheumatoid arthritis? Future Med Chem. 2015, 7(9):1137-47.

36. Aziz AUR, Farid S, Qin K, Wang H, Liu B. PIM kinases and their relevance to the PI3K/AKT/mTOR pathway in the regulation of ovarian cancer. Biomolecules. $20180204 ; 8(1)$.

37. Cravero JD, Carlson CS, Im HJ, Yammani RR, Long D, Loeser RF. Increased expression of the Akt/PKB inhibitor TRB3 in osteoarthritic chondrocytes inhibits insulin-like growth factor 1-mediated cell survival and proteoglycan synthesis. Arthritis Rheum.2009 Feb ;60(2):492-500.

38. Zhang Y, Vasheghani F, Kapoor M, et al. Cartilage-specific deletion of mTOR upregulates autophagy and protects mice from osteoarthritis. Ann Rheum Dis. 2015 Jul, 74 (7):1432-40.

39. Xue JF, Shi ZM, Zou J, Li XL. Inhibition of PI3K/AKT/mTOR signaling pathway promotes autophagy of articular chondrocytes and attenuates inflammatory response in rats with osteoarthritis. Biomed Pharmacother. 2017, 89:1252-1261.

40. Hu ZC, Gong LF, Ni WF, et al. Inhibition of PI3K/Akt/NF-kappaB signaling with leonurine for ameliorating the progression of osteoarthritis: in vitro and in vivo studies. J Cell Physiol. 2019, 234(5):6940-6950.

41. Qiu L, Zhang L, Mi X, et al. PI3K/Akt mediates expression of TNF-alpha mRNA and activation of NFkappaB in calyculin A-treated primary osteoblasts. Oral Dis. 2008, 14 (8): 727-33.

42. Chen HW, Lin AH, Liu KL, et al. Inhibition of TNF-alpha-Induced Inflammation by andrographolide via down-regulation of the PI3K/ Akt signaling pathway. J Nat Prod. 2011, 28 74(11):2408-13.

43. Liang J, Xu L, Tu M, et al. MALAT1/miR-127-5p regulates osteopontin (OPN)-mediated proliferation of human chondrocytes through PI3K/Akt pathway. J Cell Biochem. 2018, 119 (1):431-439.

44. Rao Z, Wang S, Wang J. Peroxiredoxin 4 inhibits IL-1 beta-induced chondrocyte apoptosis via PI3K/AKT signaling. Biomed Pharmacother. 2017, 90:414-420.

45. Long E, Motwani R, Seegmiller R, et al. The role of TGF-ss1 in osteoarthritis of the temporomandibular joint in two genetic mouse models. Arch Oral Biol. 2016, 67:68-73.

46. Bakker AC, van de Loo FA, van den Berg WB, et al. Overexpression of active TGF-beta-1 in the murine knee joint: evidence for synovial-layer-dependent chondroosteo- phyte formation. Osteoarthritis Cartilage. 2001, 9(2):128-36.

47. Wang W, Rigueur D, Lyons KM. TGF $\beta$ signaling in cartilage development and maintenance. Birth Defects Res C Embryo Today. 2014 Mar, 102(1):37-51.

48. Plaas A, Osborn B, Sandy JD, et al. Aggrecanolysis in human osteoarthritis: Confocal localization and biochemical characterization of ADAMTS5-hyaluronan complexes in articular cartilages. Osteoarthritis Cartilage. 2007, 15(7):719-34.

\section{Tables}


Table 1. Primers for amplification of target genes and GAPDH Forward primer sequence Reverse primer sequence

\begin{tabular}{lcc}
\hline PI3K & ACAGGCACAACGACAACATCAT & AGGTAAGCCCTAACGCAGACAT \\
AKT & TGAGACCGACACCAGGTATTTTG & GCTGAGTAGGAGAACTGGGGAAA \\
TGFß1 & CTAATGGTGGACCGCAACAAC & GTAACGCCAGGAATTGTTCTAT \\
ADAMTS-4 & GTACCTACCTGACTGGCACCATC & TGCTGCCATCTTGTCATCTGC \\
GAPDH & CTGGAGAAACCTGCCAAGTATG & GGTGGAAGAATGGGAGTTGCT \\
\hline
\end{tabular}

Table 2. Sulcus angle measurements compared between the two groups

\begin{tabular}{lccc}
\hline & Control group & Experimental group & $p$ value \\
\hline 4 weeks & $120.0^{\circ} \pm 3.0^{\circ}$ & $118.0^{\circ} \pm 2.6^{\circ}$ & 0.739 \\
8 weeks & $126.0^{\circ} \pm 3.1^{\circ}$ & $131.0^{\circ} \pm 2.9^{\circ}$ & 0.038 \\
12 weeks & $132.0^{\circ} \pm 3.4^{\circ}$ & $152.0^{\circ} \pm 3.0^{\circ}$ & 0.026 \\
\hline
\end{tabular}

mean \pm standard deviation.

Table 3. Trochlear depth measurements compared between the two groups

\begin{tabular}{lccc}
\hline & Control group & Experimental group & $p$ value \\
\hline 4 weeks & $0.5 \pm 0.1 \mathrm{~mm}$ & $0.4 \pm 0.2 \mathrm{~mm}$ & 0.913 \\
8 weeks & $0.9 \pm 0.2 \mathrm{~mm}$ & $0.6 \pm 0.2 \mathrm{~mm}$ & 0.039 \\
12 weeks & $1.5 \pm 0.2 \mathrm{~mm}$ & $0.9 \pm 0.2 \mathrm{~mm}$ & 0.029 \\
\hline
\end{tabular}

mean \pm standard deviation.

Figures 


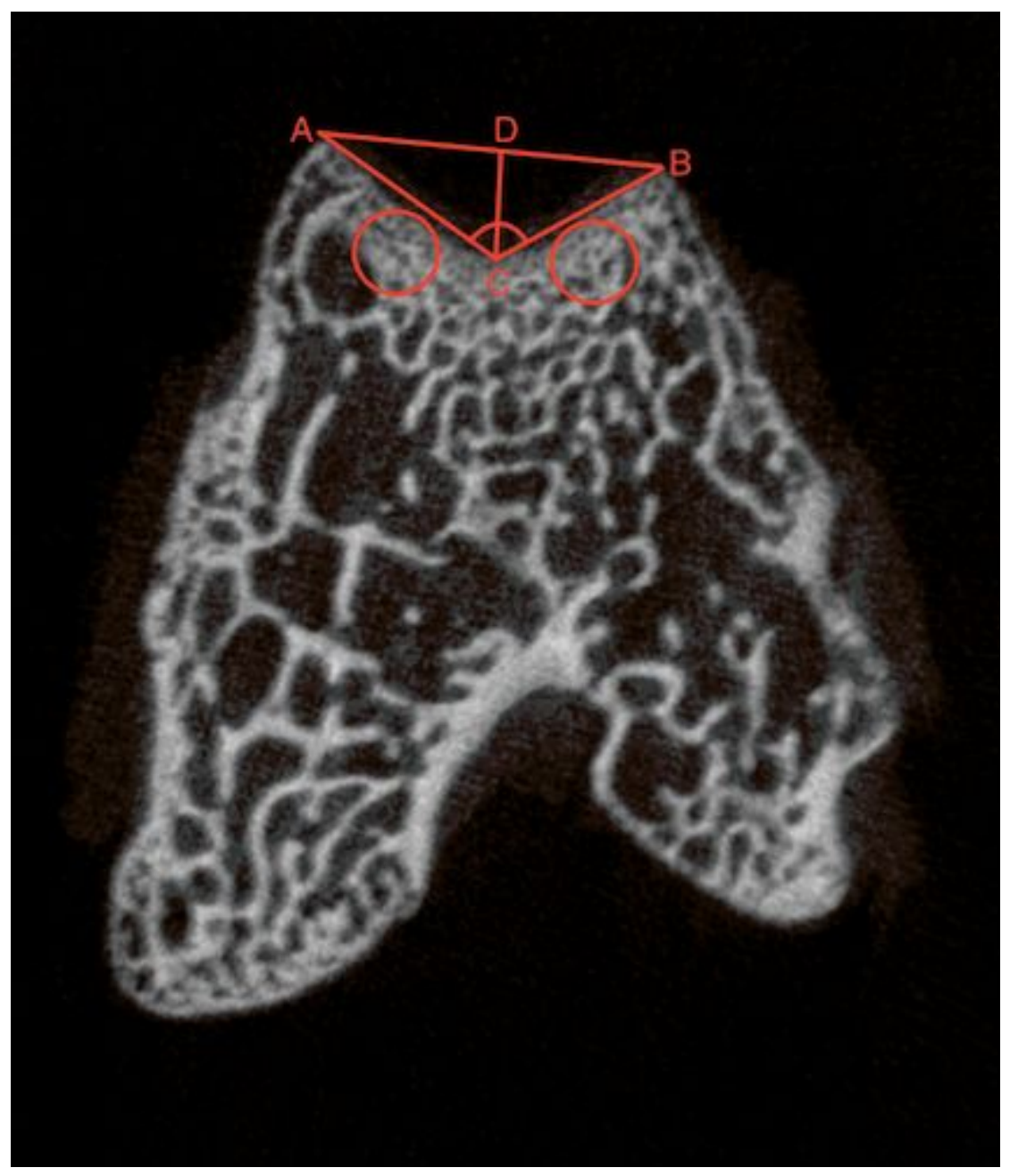

Figure 1

Measurement diagram. DC, trochlear depth; ACB, sulcus angle. 


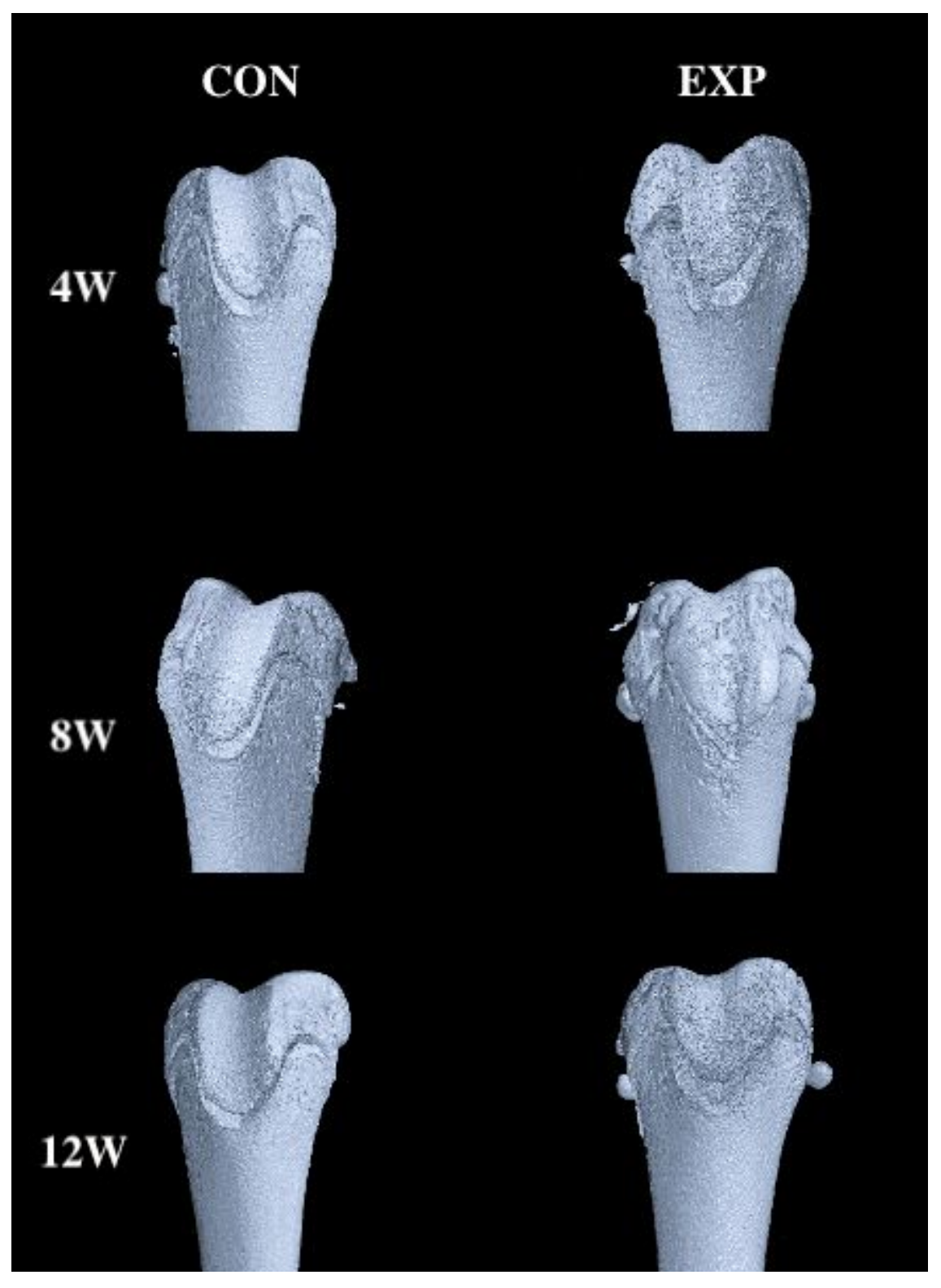

\section{Figure 2}

Three-dimensional reconstruction of distal femur by micro-computed tomography scans at 4, 8, and 12 weeks between CON and EXP groups. CON, control; EXP, experimental. 
BV/TV(\%)

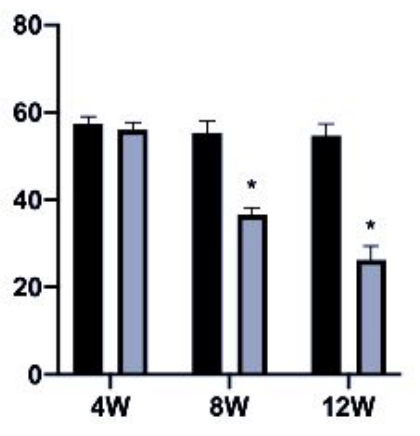

Tb.Sp(mm)

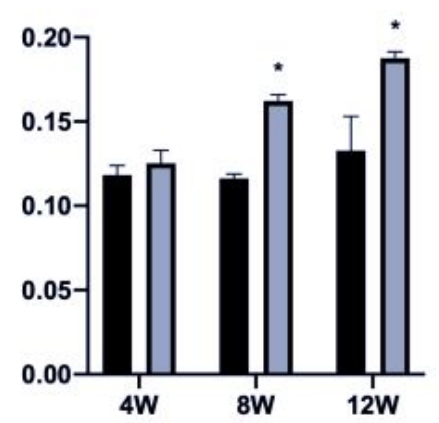

Tb.N(1/mm)

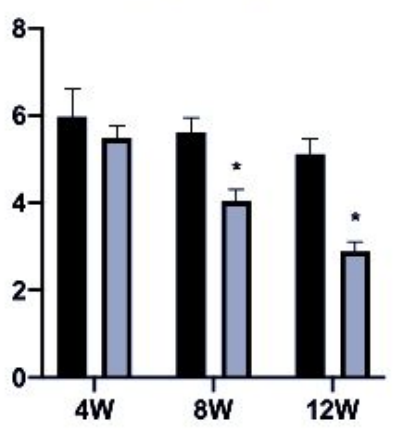

$\mathrm{BMD}\left(\mathrm{g} / \mathrm{cm}^{3}\right)$
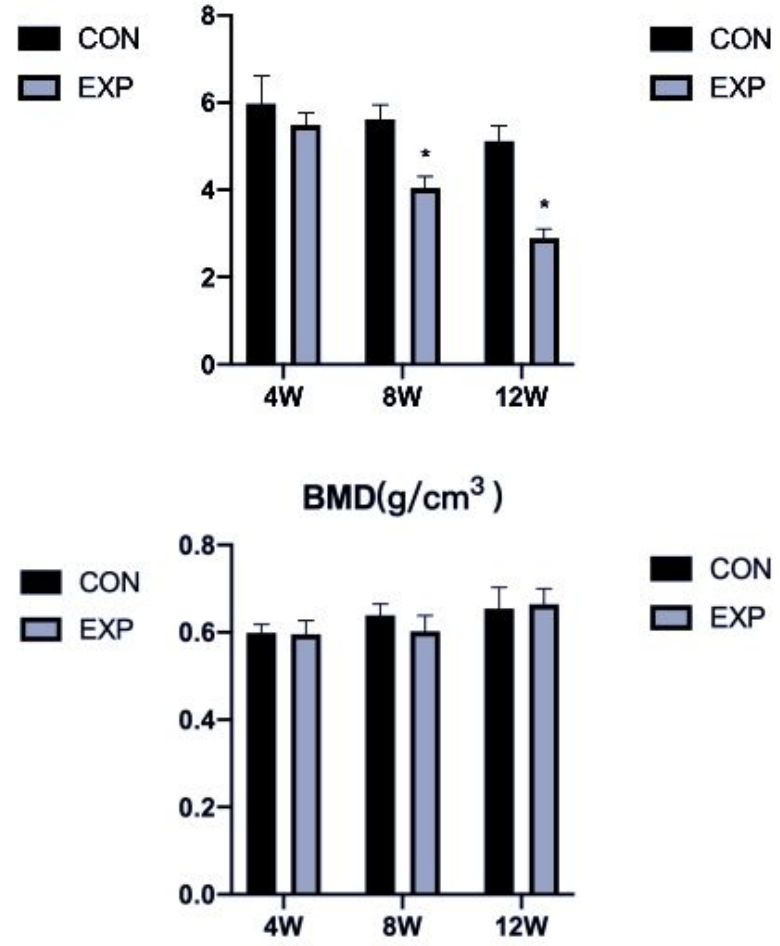

\section{Figure 3}

Histomorphometric comparison of micro-computed tomography scans between CON and EXP groups. CON, control; EXP, experimental. * $\mathrm{p}<0.05$. 

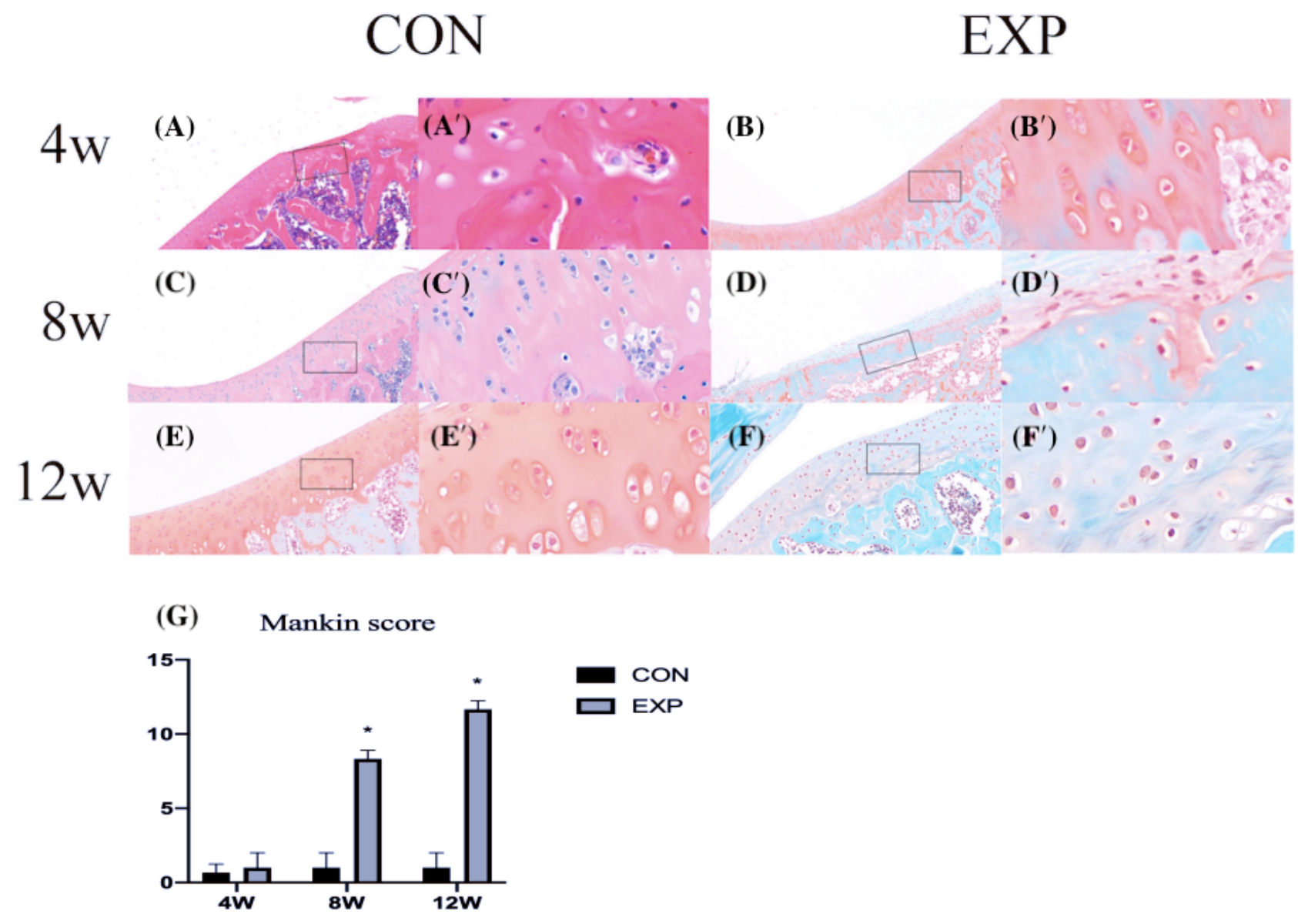

Figure 4

Safranin 0 staining in rat cartilage. CON and EXP groups, respectively: (A, B) 4 weeks; (C, D) 8 weeks; (E, F) 12 weeks. $A^{\prime}-F^{\prime}$ show higher magnifications of boxed areas in $A-F$. (G): Mankin score for trochlear cartilage. CON, control; EXP, experimental. ${ }^{*} p<0.05$.

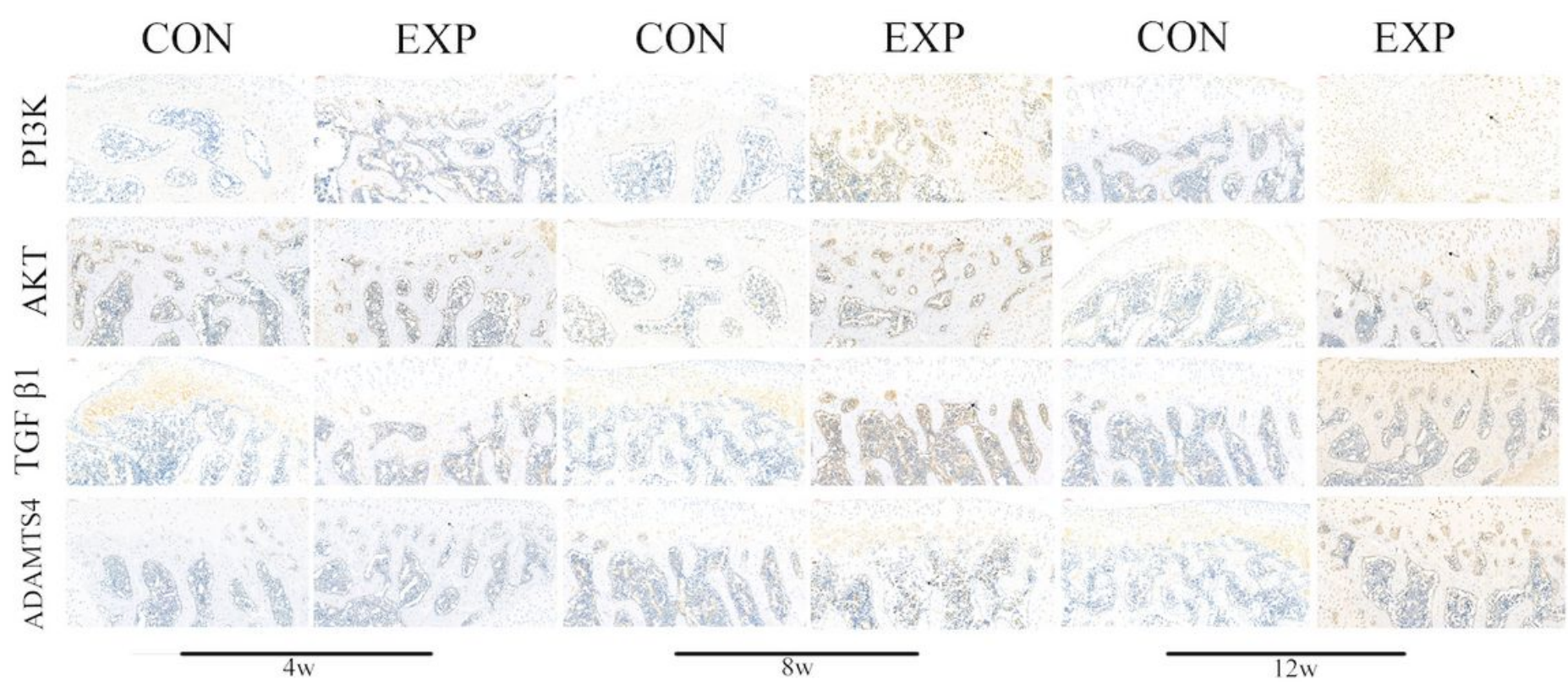


Figure 5

Immunohistochemical comparison between CON and EXP groups: PI3K/AKT, TGF $\beta 1$, and ADAMTS-4 at 4,8 , and 12 weeks. Arrows in EXP group indicate positive expression. Brown to black color indicates positive staining. Scale bar: $50 \mu \mathrm{m}$. CON, control; EXP, experimental.

PI3

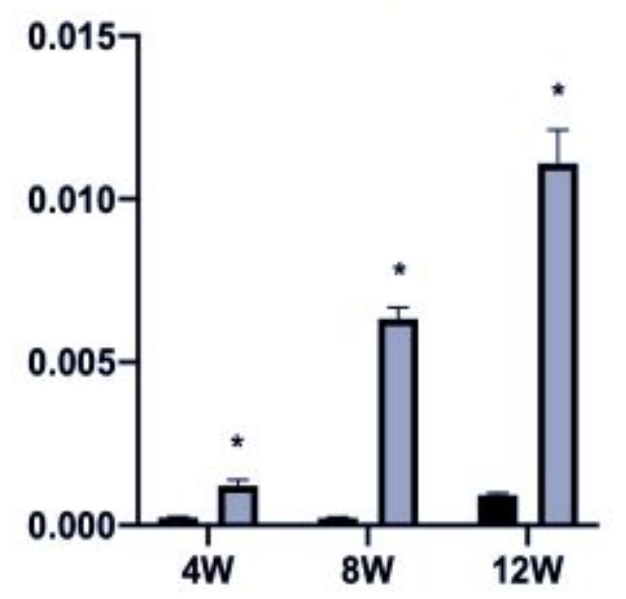

TGF $\beta 1$

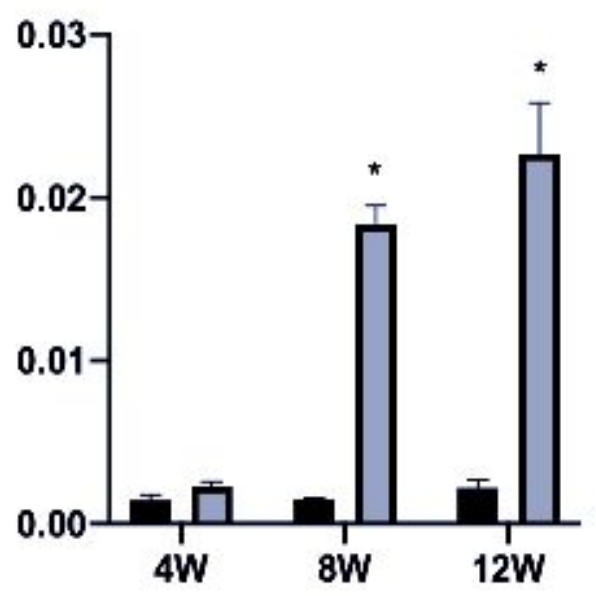

AKT

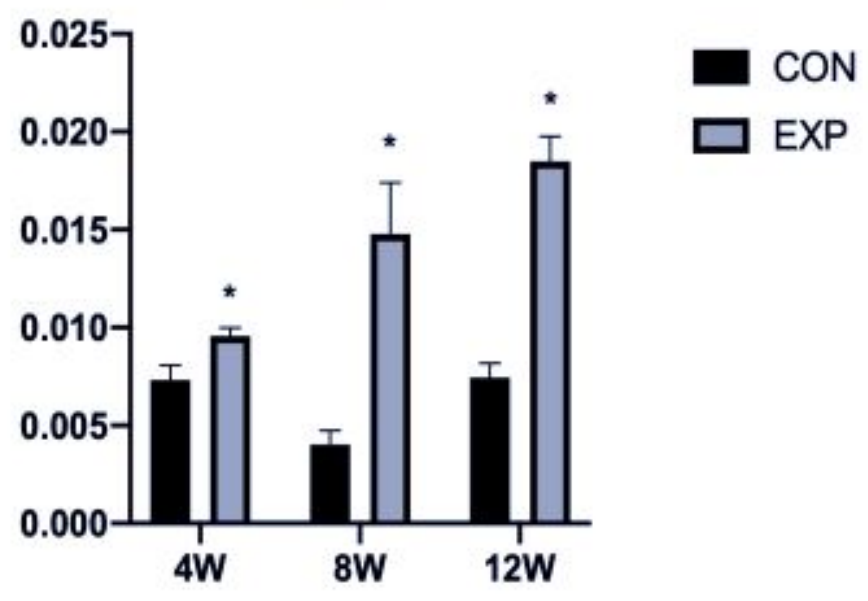

ADAMTS4

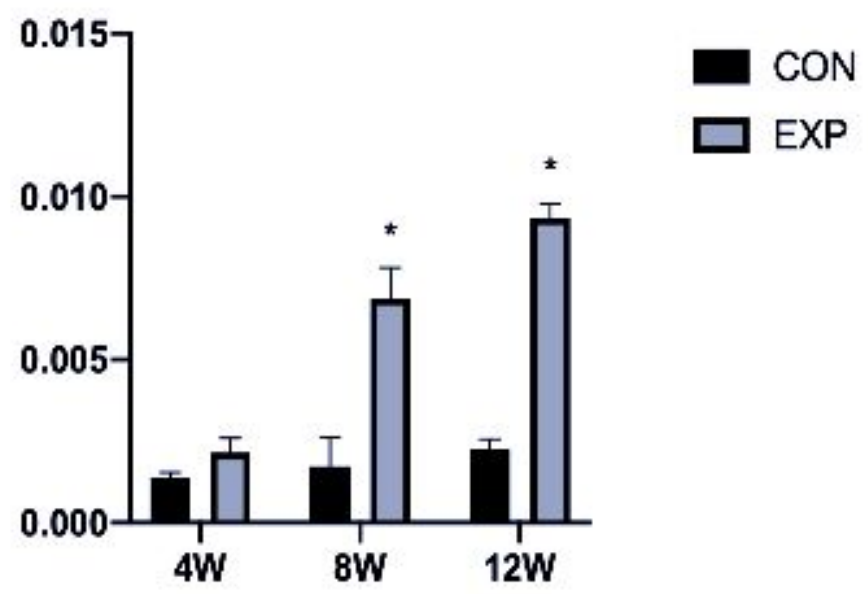

Figure 6

Positive results in immunohistochemical analysis of PI3K/AKT, TGF 31 , and ADAMTS-4 at 4, 8, and 12 weeks in CON and EXP groups. CON, control; EXP, experimental. ${ }^{*} \mathrm{p}<0.05$. 
PI3
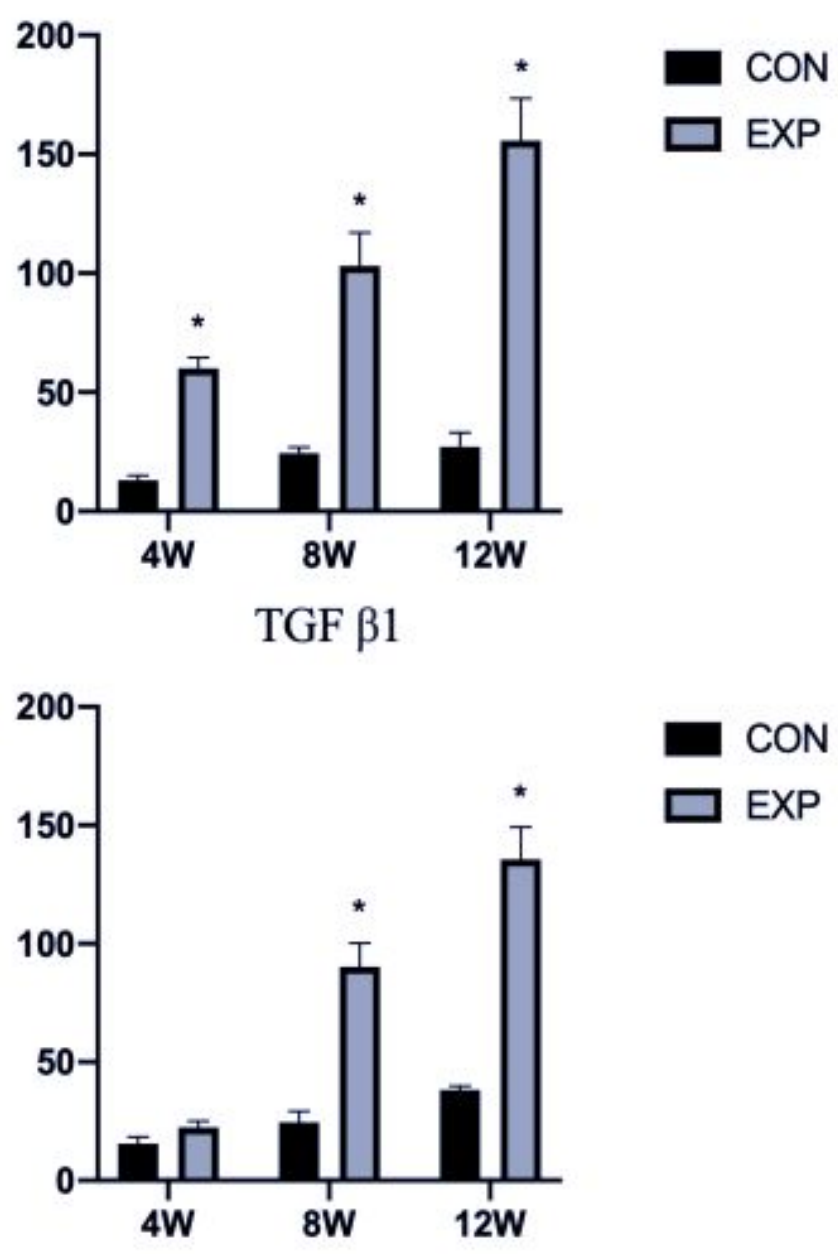

AKT
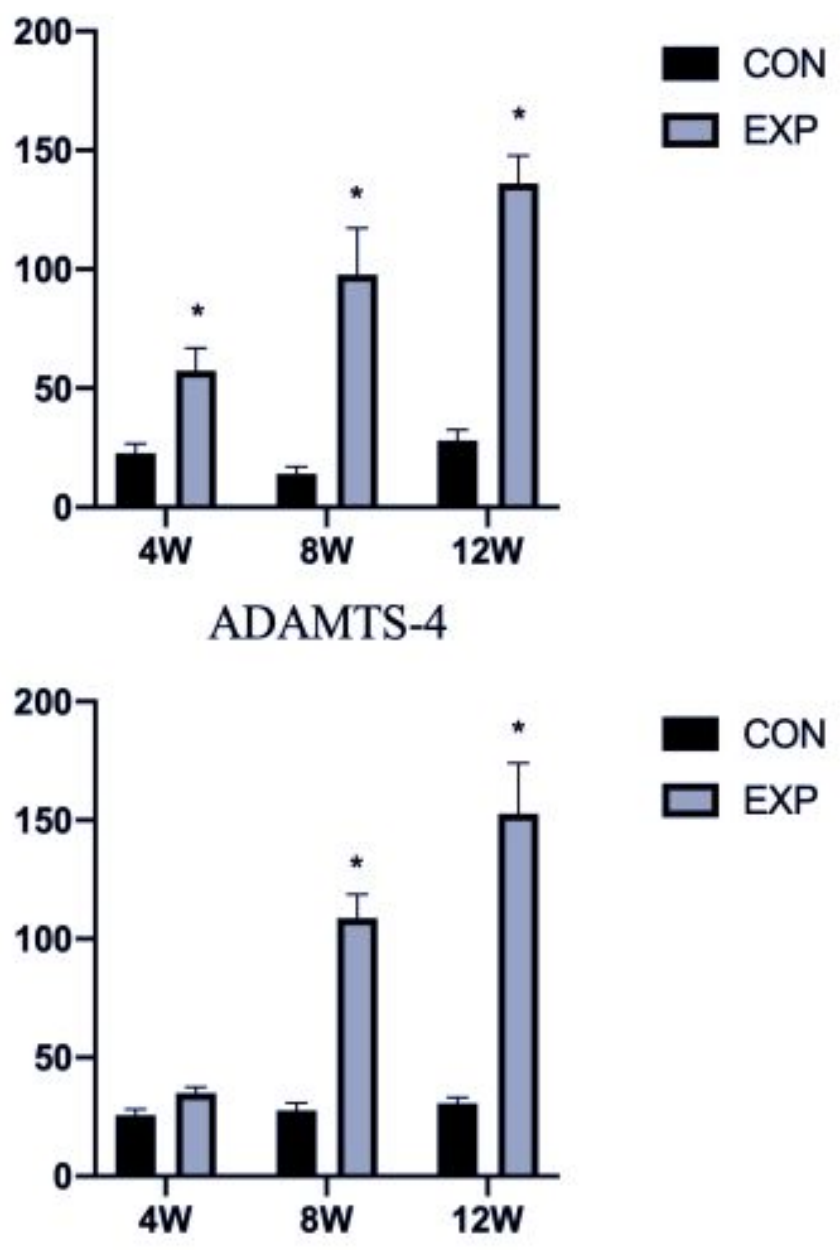

Figure 7

qPCR evaluation of PI3K/AKT, TGF $\beta 1$, and ADAMTS-4 expression levels at 4, 8, and 12 weeks in CON and EXP groups. CON, control; EXP, experimental. ${ }^{*} \mathrm{p}<0.05$. 